\title{
On the Analysis of Quasi-Static Bandpass Nonlinearities Using Volterra Series
}

\author{
Y.Shen, J.L. Tauritz \\ Telecommunication Group, University of Twente, $7500 \mathrm{AE}$ Enschede, Netherlands
}

\begin{abstract}
This paper re-examines the AM-AM and AM-PM data-based behavioral model of a narrowband quasi-static nonlinear system via Volterra series analysis. It is shown that, under narrowband input signal and flat baseband impedance assumptions, the model is in compliance with bandpass nonlinearity theory. Consequentially, there exists a relationship based on the Chebyshev-Fourier transform between the nonlinearity's one tone and two tone AM-AM transfer functions.
\end{abstract}

\section{Introduction:}

The band-pass non-linear static behavioral model can be written as:

$$
y(t)=A[r(t)] \cos \left(\omega_{0} t+\varphi(t)+\Phi[r(t)]\right)
$$

with input signal:

$$
x(t)=r(t) \cos \left[\omega_{0} t+\varphi(t)\right]
$$

$A(r)$ and $\Phi(r)$ in (1) are the envelope and phase transfer function of the band-pass nonlinearity, respectively. For a known instantaneous nonlinearity, $A(r)$ is obtained by Chebyshev transforming that nonlinearity ${ }^{[1][2]}$. Good simulation results have been achieved using this model ${ }^{[2]}$.

In practical applications when the instantaneous nonlinearity is unknown, $\mathrm{A}(\mathrm{r})$ and $\Phi(r)$ are normally derived by fitting the AM-AM and AM-PM data from either one tone measurement or circuit level simulation ${ }^{[3]}$. Measurement data collected in this way has been shown to be quite effective in characterizing the nonlinearity compared to the results from circuit level simulations, in cases where thermal and DC bias effects are negligible ${ }^{[4][5]}$. Nevertheless, the theoretical basis for and the limits on the validity of the model are somewhat vague.

This paper investigates this AM-AM and AM-PM data based model by means of Volterra series analysis. To do so, the fitted nonlinearity of the one tone AM-AM data is treated as an instantaneous nonlinearity. Then, it is shown that when specific assumptions are satisfied, the Chebyshev Fourier transformation that relates the instantaneous non-linearity and its AM-AM fitted nonlinearity, is the two tone envelope AM-AM transfer function. This establishes the use of one tone AM-AM and AM-PM data for modelling band-pass nonlinearities. Finally, we show that the necessary conditions for the assumptions to be met are that the envelope signal is narrowband and that the base-band impedance is flat over the bandwidth of the envelope signal.

\section{Two tone envelope AM-AM transfer function}


When referring to the bandpass nonlinearity the nonlinearity is taken to be centred on the carrier frequency, which can also be seen as an instantaneous nonlinearity itself. Fig. 1 depicts these two non-linearities.

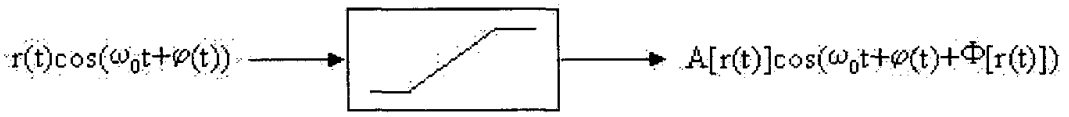

(a) Banchass nonlinearity of the envelope signal

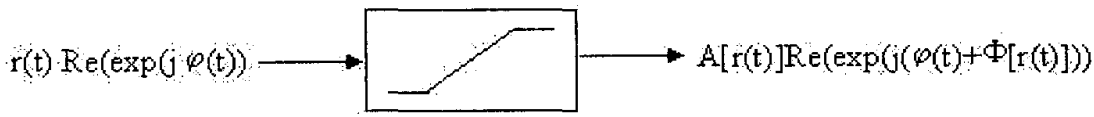

(b) Instantaneous nonlinearity of the envel ope signal

Fig.1 Bandpass nonlinearity and instantaneous nonlinearity

For an instantaneous nonlinearity as shown in fig.1-b, its envelope one tone AMAM data can be obtained using two tone input measurements or simulation. This is illustrated in fig. 2 and equation (3).

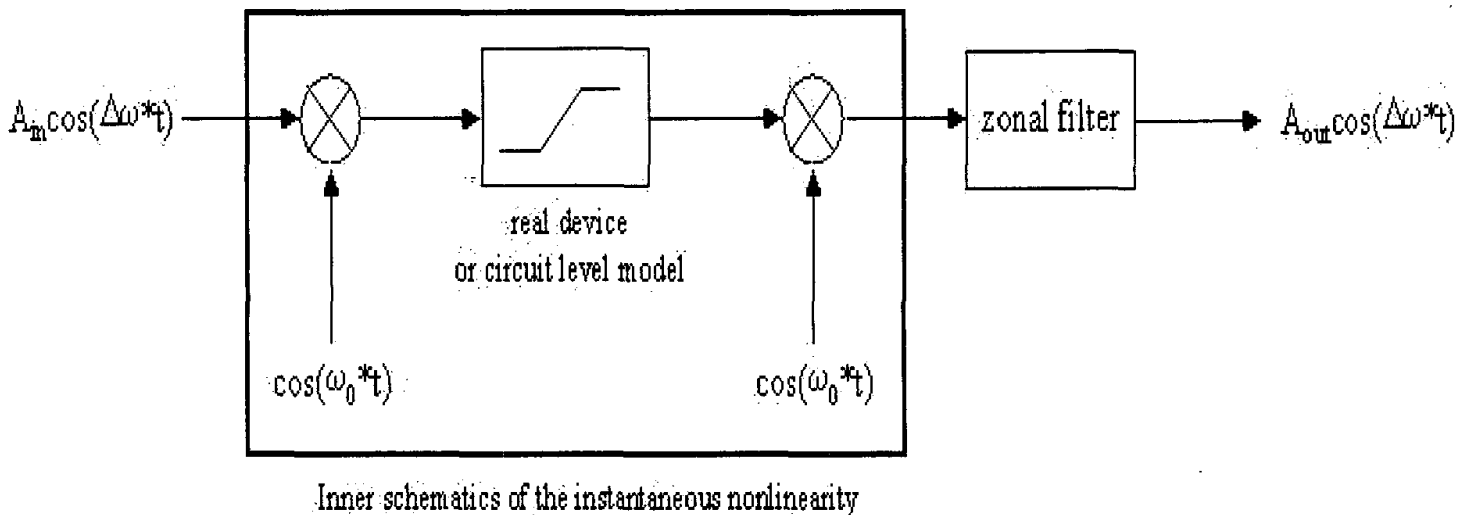

Fig.2 Virtual envelope one tone measurement

$$
A_{\text {in }} \cos (\Delta \omega t) * \cos \left(\omega_{0} t\right)=\frac{A_{\text {in }}}{2} \cos \left(\omega_{1} t\right)+\frac{A_{\text {in }}}{2} \cos \left(\omega_{2} t\right)
$$

where

$$
\omega_{1}=\omega_{0}-\Delta \omega \quad \omega_{2}=\omega_{0}+\Delta \omega
$$

In light of bandpass nonlinearity theory, a Chebyshev Fourier transformation relationship exists between the instantaneous nonlinearity and the bandpass nonlinearity. In the following section this relationship is observed, using Volterra series analysis, between the one tone AM-AM data and the envelope AM-AM data.

\section{Volterra series analysis}


We consider a system that can be modelled by the third order Volterra series. The system's response to a single input tone $x(t)=A \cos \left(2 \pi f_{0} t\right)$ is: ${ }^{[6]}$

$$
y(t)=\cos \left(2 \pi f_{0} t\right)\left[\frac{A}{2} H_{1}+\frac{A^{3}}{16} H_{3} \cos \alpha+j \frac{A^{3}}{16} H_{3} \sin \alpha\right]
$$

where

$$
H_{1}=\operatorname{Re}\left\{\tilde{H}_{1}\left(f_{0}\right)\right\}, H_{3}=\operatorname{Re}\left\{\tilde{H}_{3}\left(f_{0}, f_{0},-f_{0}\right)\right\}, \quad \alpha=\operatorname{angle}\left(\frac{\tilde{H}_{3}\left(f_{0}, f_{0},-f_{0}\right)}{\tilde{H}_{1}\left(f_{0}\right)}\right)
$$

and $\tilde{H}_{1}(f), \tilde{H}_{3}\left(f_{a}, f_{b}, f_{c}\right)$ are the system's first and third order Volterra nonlinear transfer functions.

The system's response to a two tone input signal

$$
x(t)=\frac{A}{2}\left[\cos \left(2 \pi f_{1} t\right)+\cos \left(2 \pi f_{2} t\right)\right]=A \cos (2 \pi \Delta f t) \cos \left(2 \pi f_{0} t\right)
$$

is given by:

$$
\begin{aligned}
y(t) & =\frac{1}{2}\left\{\cos \left(2 \pi f_{1} t\right)\left[\frac{A}{2} \tilde{H}_{1}\left(f_{1}\right)+\frac{A^{3}}{64} \tilde{H}_{3}\left(f_{1}, f_{1},-f_{1}\right)+\frac{A^{3}}{32} \tilde{H}_{3}\left(f_{1}, f_{2},-f_{2}\right)\right]\right. \\
& +\cos \left(2 \pi f_{2} t\right)\left[\frac{A}{2} \tilde{H}_{1}\left(f_{2}\right)+\frac{A^{3}}{64} \tilde{H}_{3}\left(f_{2}, f_{2},-f_{2}\right)+\frac{A^{3}}{32} \tilde{H}_{3}\left(f_{2}, f_{1},-f_{1}\right)\right] \\
& +\cos \left[2 \pi\left(2 f_{2}-f_{1}\right) t\right]\left[\frac{A^{3}}{64} \tilde{H}_{3}\left(-f_{1}, f_{2}, f_{2}\right)\right] \\
& \left.+\cos \left[2 \pi\left(2 f_{1}-f_{2}\right) t\right]\left[\frac{A^{3}}{64} \tilde{H}_{3}\left(-f_{2}, f_{1}, f_{1}\right)\right]\right\}
\end{aligned}
$$

Since we are talking about narrowband situations, we have

$$
f_{1} \cong f_{0} \cong f_{2}
$$

let us assume that:

$$
\begin{gathered}
\tilde{H}_{1}\left(f_{1}\right)=\tilde{H}_{1}\left(f_{0}\right)=\tilde{H}_{1}\left(f_{2}\right) \\
\tilde{H}_{3}\left(f_{i}, f_{i},-f_{i}\right)=\tilde{H}_{3}\left(f_{0}, f_{0},-f_{0}\right) \quad i=1 \text { or } 2
\end{gathered}
$$

and

$$
\tilde{H}_{3}\left(f_{i}, f_{j},-f_{j}\right)=\tilde{H}_{3}\left(f_{0}, f_{0},-f_{0}\right) \quad i=1, j=2 \quad \text { or } \quad i=2, j=1
$$

then (6) can be simplified to 


$$
\begin{aligned}
y(t)= & \cos (2 \pi \Delta f t) \cos \left(2 \pi f_{c} t\right)\left[\frac{A}{2} H_{1}+\frac{3 A^{3}}{64} H_{3} \cos \alpha+j \frac{3 A^{3}}{64} H_{3} \sin \alpha\right] \\
& +\cos \left(2 \pi\left(2 f_{2}-f_{1}\right) t\right)\left[\frac{A^{3}}{64} \tilde{H}_{3}\left(-f_{1}, f_{2}, f_{2}\right)\right] \\
& +\cos \left(2 \pi\left(2 f_{1}-f_{2}\right) t\right)\left[\frac{A^{3}}{64} \tilde{H}_{3}\left(-f_{2}, f_{1}, f_{1}\right)\right]
\end{aligned}
$$

We will examine the validity of assumptions (8) (9) and (10) in the next section. In this section the assumptions are deemed appropriate. From (4), we see that the AM$\mathrm{AM}$ characteristic for a single input tone is:

$$
G(A)=\sqrt{\frac{A^{2}}{4} H_{1}{ }^{2}+\frac{A^{6}}{256} H_{3}{ }^{2}+\frac{A^{4}}{16} H_{1} H_{3} \cos \alpha}
$$

while from (10), the AM-AM characteristic for the envelope signal with the same amplitude for the single tone input is:

$$
g(A)=\sqrt{\frac{A^{2}}{4} H_{1}^{2}+\frac{9 A^{6}}{4096} H_{3}^{2}+\frac{3 A^{4}}{64} H_{1} H_{3} \cos \alpha} .
$$

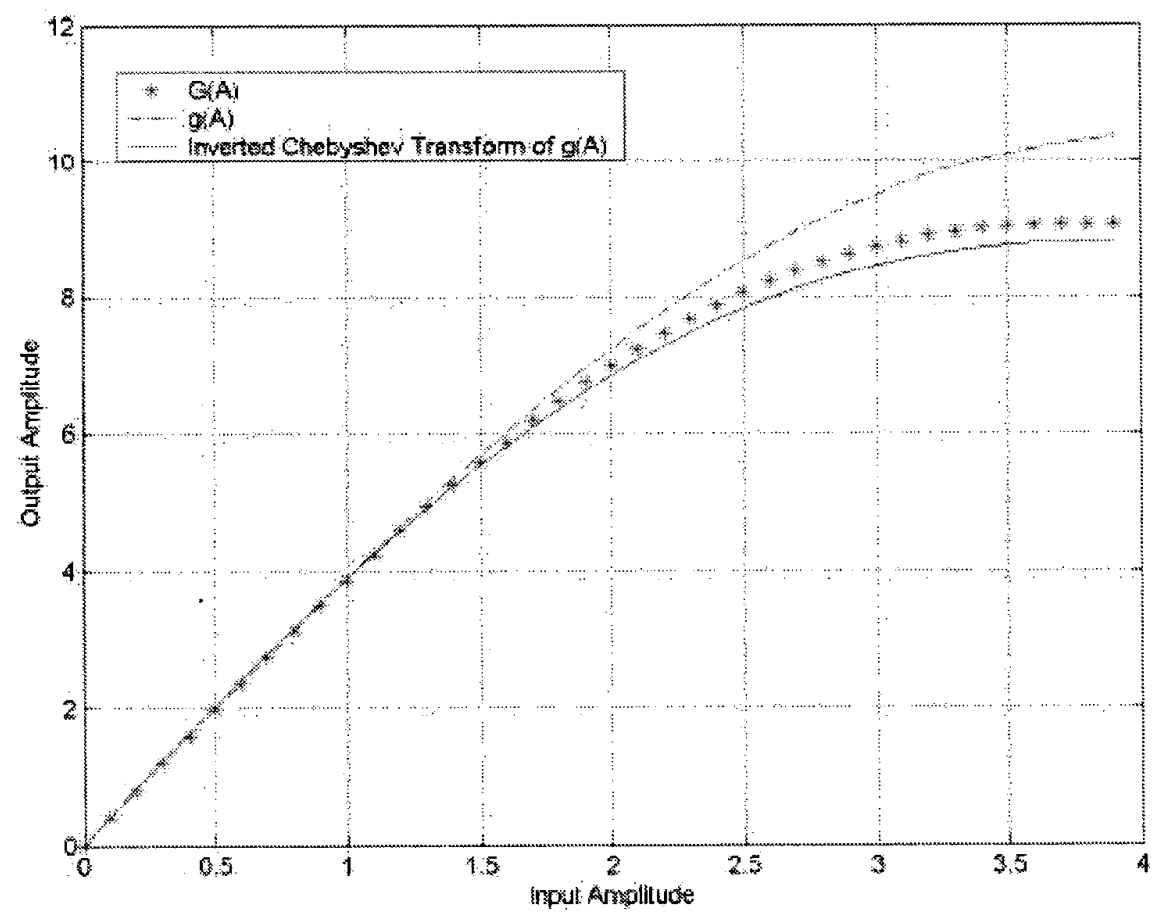

(a) $\mathrm{H}_{3} / \mathrm{H}_{1}=0.3, \alpha=5 \pi / 6$ 


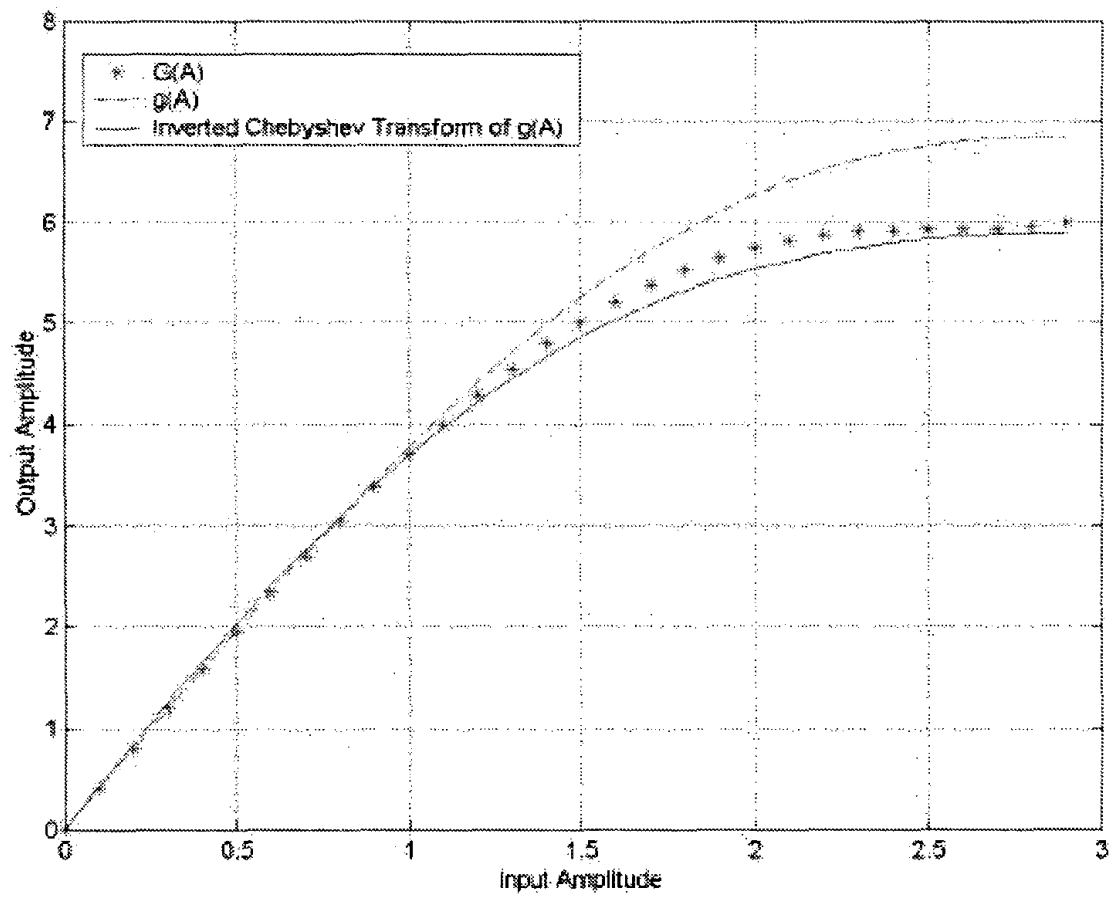

(b) $\mathrm{H}_{3} / \mathrm{H}_{1}=0.7, \alpha=5 \pi / 6$

Fig.3 Comparison of $G(A), g(A)$ and the Inverted Chebyshev Transformation of $g(A)$

Fig.3 illustrates the relationship of the Chebyshev Fourier transformation between $\mathrm{G}(\mathrm{A})$ and $\mathrm{g}(\mathrm{A})$. According to the theory of bandpass nonlinearity, it suggests that the AM-AM characteristic of the one tone signal is the instantaneous nonlinear transfer function of the envelope AM-AM data. Therefore, if (8) (9) and (10) are met, the AM-AM and AM-PM data fitted transfer functions are representative of the band-pass non-linearity of the envelope signal.

\section{Assumptions examined}

In order to investigate under what conditions assumptions (8) (9) and (10) can be met, we take advantage of the closed form of the Volterra non-linear transfer functions for the general non-linear circuit shown in fig. $4^{[7]}$,

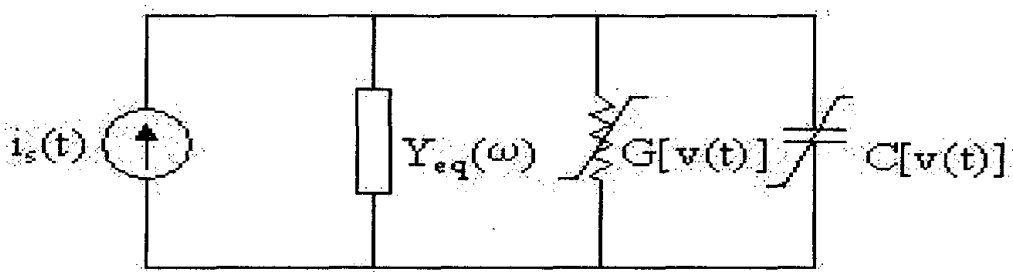

Fig.4 A general nonlinear circuit 


$$
\begin{aligned}
& H_{1}(\omega)=\frac{1}{Y_{e q}(\omega)}=Z_{e q}(\omega) \\
& H_{2}\left(\omega_{1}, \omega_{2}\right)=-Z_{e q}\left(\omega_{1}+\omega_{2}\right)\left[G_{2}+j\left(\omega_{1}+\omega_{2}\right) C_{2}\right]\left[H_{1}\left(\omega_{1}\right) H_{1}\left(\omega_{2}\right)\right] \\
& H_{3}\left(\omega_{1}, \omega_{2}, \omega_{3}\right)=\frac{-Z_{e q}\left(\omega_{1}+\omega_{2}+\omega_{3}\right)}{6}\left\{4\left[G_{2}+j\left(\omega_{1}+\omega_{2}+\omega_{3}\right) C_{2}\right]\right. \\
& \quad\left[H_{1}\left(\omega_{1}\right) H_{2}\left(\omega_{2}, \omega_{3}\right)+H_{1}\left(\omega_{2}\right) H_{2}\left(\omega_{1}, \omega_{3}\right)+H_{1}\left(\omega_{3}\right) H_{2}\left(\omega_{1}, \omega_{2}\right)\right]+ \\
& \left.6\left[G_{3}+j\left(\omega_{1}+\omega_{2}+\omega_{3}\right) C_{3}\right]\left[H_{1}\left(\omega_{1}\right) H_{1}\left(\omega_{2}\right) H_{1}\left(\omega_{3}\right)\right]\right\}
\end{aligned}
$$

Substituting

$$
\begin{aligned}
& H_{1}\left(\omega_{i}\right)=\frac{1}{Y_{e q}\left(\omega_{i}\right)}=Z_{e q}\left(\omega_{i}\right) \quad i=0,1 \quad \text { or } 2 \\
& H_{2}\left(\omega_{i}, \omega_{i}\right)=-Z_{e q}\left(2 \omega_{i}\right)\left[G_{2}+j\left(2 \omega_{i}\right) C_{2}\right] H_{1}^{2}\left(\omega_{i}\right) \quad i=0, \quad 1 \quad \text { or } \\
& H_{2}\left(\omega_{i},-\omega_{i}\right)=-Z_{e q}(0) G_{2} H_{1}\left(\omega_{i}\right) H_{1}\left(-\omega_{i}\right) \quad i=0, \quad 1 \quad \text { or } \\
& H_{2}\left(\omega_{i},-\omega_{j}\right)=-Z_{e q}\left(\omega_{i}-\omega_{j}\right)\left[G_{2}+j\left(\omega_{i}-\omega_{j}\right) C_{2}\right] H_{1}\left(\omega_{i}\right) H_{1}\left(-\omega_{j}\right) \\
& H_{3}\left(\omega_{i}, \omega_{i},-\omega_{i}\right)=\frac{-Z_{e q}\left(\omega_{i}\right)}{6}\left\{4 [ G _ { 2 } + j \omega _ { i } C _ { 2 } ] \left[2 H_{1}\left(\omega_{i}\right) H_{2}\left(\omega_{i},-\omega_{i}\right)+\right.\right. \\
& \left.\left.\quad H_{1}\left(-\omega_{i}\right) H_{2}\left(\omega_{i}, \omega_{i}\right)\right]+6\left[G_{3}+j \omega_{i} C_{3}\right] H_{1}^{2}\left(\omega_{i}\right) H_{1}\left(-\omega_{i}\right)\right\} \\
& H_{3}\left(\omega_{i}, \omega_{j},-\omega_{j}\right)=\frac{-Z_{e q}\left(\omega_{i}\right)}{6}\left\{4\left[G_{2}+j \omega_{i} C_{2}\right]\right. \\
& {\left[H_{1}\left(\omega_{i}\right) H_{2}\left(\omega_{j},-\omega_{j}\right)+H_{1}\left(\omega_{j}\right) H_{2}\left(\omega_{i},-\omega_{j}\right)+H_{1}\left(-\omega_{j}\right) H_{2}\left(\omega_{i}, \omega_{j}\right)\right]+} \\
& \left.6\left[G_{3}+j \omega_{i} C_{3}\right]\left[H_{1}\left(\omega_{i}\right) H_{1}\left(\omega_{j}\right) H_{1}\left(\omega_{-j}\right)\right]\right\} \\
& i=1, \quad j=2 \quad \text { or }
\end{aligned}
$$

It is easily seen that in the narrowband case assumptions (8) and (9) are satisfied. However, if the baseband impedance is not flat assumption (10) is not satisfied. This is shown in equation (23) assuming a narrowband input:

$$
\begin{aligned}
& H_{3}\left(\omega_{i}, \omega_{j},-\omega_{j}\right)-H_{3}\left(\omega_{0}, \omega_{0},-\omega_{0}\right) \cong \\
& \frac{-Z_{e q}^{2}\left(\omega_{0}\right)}{6}\left\{4\left[G_{2}+j \omega_{0} C_{2}\right]\left[Z_{e q}(0)-Z_{e q}\left(\omega_{i}-\omega_{j}\right)\right] H_{1}\left(\omega_{0}\right) H_{1}\left(-\omega_{0}\right)\right. \\
& i=1, \quad j=2 \quad \text { or } \quad i=2, \quad j=1
\end{aligned}
$$


Equation (23) is illustrative of the considerable influence of sub-harmonic frequency terminations and bias circuits on the model's accuracy, a phenomenon previously reported ${ }^{[8]}$.

In order to test our theoretical work, we considered a realistic power amplifier using a harmonic balance based simulator. The result is shown in fig.3.



Fig.5 Comparison of $\mathrm{G}(\mathrm{A}), \mathrm{g}(\mathrm{A})$ and $\mathrm{g}(\mathrm{A})$ 's Chebyshev Transformation with data from $\mathrm{HB}$ simulation

\section{Conclusions}

This paper demonstrates that when the envelope signal is narrowband and the baseband impedance of the circuit is flat, the two tone or envelope AM-AM data correlates well with the Chebyshev transformed one tone measured or simulated AMAM data. Thus, consistent with the theory of bandpass nonlinearity, the one tone measured or simulated AM-AM and AM-PM data can be used as the bandpass quasistatic non-linear behavioural model.

\section{Acknowledgements} EEsof.

This work was supported by Philips Semiconductors Nijmegen and Agilent-

\section{References:}


[1] N. M. Blachman, "Detectors, Bandpass Nonlinearities, and Their Optimization: Inversion of the Chebyshev Transform", IEEE Trans. on Information Theory, vol 17, Jul. 1971, pp. 398-404.

[2] A. R. Kaye, D. A. George, "Analysis and Compensation of Bandpass

Nonlinearities for Communications," IEEE Trans. Commun., Oct. 1972, pp. 965972.

[3] Adel A. M. Saleh, "Frequency-Independent and Frequency-Dependent Nonlinear Models of TWT Amplifiers", IEEE Trans. Commun., Nov. 1981, pp. 1715-1720.

[4] J. Staudinger, "Applying the Quadrature Modeling Technique to Wireless Power Amplifiers," Microwave Journal, Nov. 1997, pp. 66-86.

[5] Reza Mahmoudi, J. L. Tauritz, "Multi-disciplinary Design Method for Second and Third Generation Mobile Communication Systems", IEEE MTT-S 2001, Workshop Dynamics of the Microwave Workbench, Phoenix, May 2001.

[6] A. Leke, J.S. Kenney, "Behavioral Modeling of Narrowband Microwave Power Amplifiers with Applications in Simulating Spectral Regrowth," IEEE MTT-S 1996, pp. 1385-88.

[7] N. B. Carvalho, J. C. Pedro,"Two-Tone IMD Asymmetry in Microwave Power Amplifiers," IEEE MTT-S 2000, Boston.

[8] J. Staudinger, "The Importance of Sub-Harmonic Frequency Terminations in Modeling Spectral Regrowth from CW AM-AM \& AM-PM Derived NonLinearities," 1997 Wireless Communications Conference, pp. 121-125. 